\title{
Anomalous thermal expansion and luminescence thermochromism in silver(I) dicyanamide
}

\author{
Sarah A. Hodgson, ${ }^{[a]}$ Sarah J. Hunt, ${ }^{[a]}$ Thomas Just Sørensen, ${ }^{* a, b]}$ Amber L. Thompson, ${ }^{[a]}$ \\ Emily M. Reynolds, ${ }^{[a]}$ Stephen Faulkner, ${ }^{[a]}$ and Andrew L. Goodwin*[a]
}

Abstract: We study mechanical flexibility and its link to optical properties in the dense metal-organic framework silver(I) dicyanamide, $\operatorname{Ag}\left[\mathrm{N}(\mathrm{CN})_{2}\right]$. We show that the system exhibits polymorph-independent anomalous thermal expansion behaviour and find a linear variation in the relative intensity of emission bands with temperature, indicative of thermochromism in the Ag-centered luminescence.

\section{Introduction}

One of the most appealing aspects of metal-organic frameworks (MOFs) is their mechanical flexibility. ${ }^{[1]}$ Their open structuresassembled from hinge-like nodes connected by long, flexible linkers-allow MOFs to exhibit large structural responses to external stimuli. ${ }^{[2]}$ This flexibility has been exploited in, for example, solvent-responsive "breathing" in transition-metal dicarboxylates, ${ }^{[3]}$ methane storage,$^{[4]}$ colossal negative thermal expansion, ${ }^{[5]}$ negative compressibility ${ }^{[6]}$ and ferroelasticity. ${ }^{[7]}$ One of the clearest challenges in the field is to link this strong mechanical response to other physical properties that depend strongly on distance. In this respect, optical response is an obvious candidate, potentially allowing the exploitation of mechanical flexibility in high-performance sensors.

It was in this context that we chose to study the effect of temperature on the structure and luminescent behaviour of silver(I) dicyanamide, Ag(dca). ${ }^{[8]}$ Under ambient conditions, $\mathrm{Ag}(\mathrm{dca})$ can be prepared in two structurally-related polymorphs, one with trigonal $P 3_{1} 21 / P 3_{2} 21$ symmetry and one with orthorhombic Pnma symmetry. The structures of both consist of a triangular array of 1D polymer chains. In the case of the trigonal polymorph these chains form triangular helices of a single handedness [Fig. 1(a)] whereas in the orthorhombic polymorph the chains are achiral "zig-zags" [Fig. 1(b)]. A combination of argentophilic and weak electrostatic interactions connect the chains along directions perpendicular to the chain axes. These weaker interactions describe complex threedimensional nets that in the case of trigonal $\mathrm{Ag}(\mathrm{dca})$ relate its

\footnotetext{
[a] S. A. Hodgson, S. J. Hunt, T. J. Sørensen, A. L. Thompson, E. M. Reynolds, S. Faulkner, A. L. Goodwin Department of Chemistry

University of Oxford Inorganic Chemistry Laboratory, South Parks Road, Oxford OX1 3QR, U.K.

E-mail: tjs@chem.ku.dk or andrew.goodwin@chem.ox.ac.uk

[b] T. J. Sørensen Nano-Science Center and Department of Chemistry University of Copenhagen Universitetsparken 5

2100 København $\varnothing$, Denmark
}

structure to that of $\alpha$-quartz. ${ }^{[9]}$

We selected this particular system for two specific reasons. On the one hand, helical structural motifs are known to promote mechanical flexibility: ${ }^{[10,11]}$ on the other hand, the argentophilic interactions that mediate extreme mechanical response are precisely those with strong distance-dependent luminescent behaviour. ${ }^{[12]}$ So our approach is straightforward. We first establish the extent and nature of mechanical flexibility in $\mathrm{Ag}$ (dca) by measuring its structural response to temperature. Then we demonstrate its luminescence thermochromism by measuring its spectroscopic response as a function of temperature.

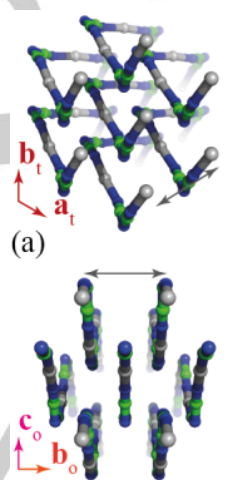

(b)

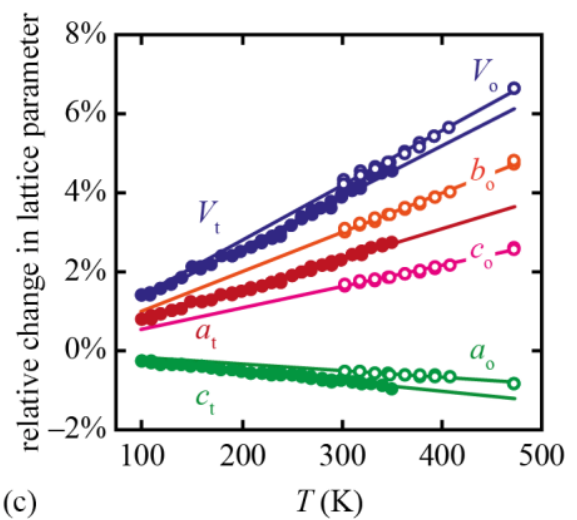

(c)

$T(\mathrm{~K})$
Figure 1. Representation of the crystal structures of (a) trigonal and (b) orthorhombic modifications of $\mathrm{Ag}(\mathrm{dca})$. $\mathrm{Ag}$ atoms in silver; $\mathrm{C}$ in green and $\mathrm{N}$ in blue; argentophilic interactions are indicated by arrows. (c) Relative change in unit cell dimensions determined using single-crystal X-ray diffraction (trigonal polymorph, filled symbols) and powder X-ray diffraction (orthorhombic polymorph, open symbols). Lines represent fits. In both cases negative thermal expansion (NTE) occurs along the chain axis (trigonal $c$, orthorhombic a) and positive thermal expansion (PTE) is strongest in the directions of argentophilic Ag....Ag interactions (trigonal $a$, orthorhombic $b$ ).

\section{Results and Discussion}

Samples of both polymorphs of $\mathrm{Ag}(\mathrm{dca})$ were prepared using a combination of slow diffusion and rapid precipitation methods. Slow diffusion yielded the trigonal polymorph as single crystals; these grew as merohedral twins in enantiomorphic space groups $P 3_{1} 21 / P 3_{2} 21$, as determined by single crystal X-ray diffraction measurements. ${ }^{[(a)]}$ Rapid precipitation yielded the orthorhombic polymorph as a polycrystalline powder sample; its structure was found to be consistent with previous reports. ${ }^{[(b)}$ )]

The thermal expansion properties of both phases were determined using variable-temperature X-ray diffraction measurements. We found no evidence for structural phase 
transitions in either polymorph over the temperature ranges investigated. The temperature dependence of the lattice parameters is illustrated in Fig. 1(c), from which it is clear that both polymorphs show similar thermal responses. On cooling, the chains pack more densely and become increasingly linear, the latter effect requiring elongation of the chain axis. This negative thermal expansion (NTE) response is conceptually similar to that reported for the low-temperature form of $\mathrm{Cu}(\mathrm{CN})^{[13]}$ and is balanced by strong positive thermal expansion (PTE) in directions perpendicular to the chain axis. Note that the PTE is strongest in the directions of argentophilic $\mathrm{Ag}$... Ag interactions (trigonal $a$, orthorhombic $b$ ). Thermal expansion is quantified by the expansivities $(\alpha=\Delta / / I \Delta T)$, which our data give to be $\alpha_{a}=-16.7(8), \alpha_{b}=97(3)$, and $\alpha_{c}=53.0(1.9) \mathrm{MK}^{-1}$ for the orthorhombic polymorph and $\alpha_{a}=76.5(8)$ and $\alpha_{c}=-25.5(5) \mathrm{MK}^{-1}$ for the trigonal polymorph. ${ }^{[14]}$ The PTE behaviour we observe is strong with respect to typical responses of solid materials ( $\alpha$ 0-20 MK ${ }^{-1}$ ), but is representative of flexible MOF-type systems where mechanical response is governed by metallophilic interactions. ${ }^{[15]}$ The similarity in supramolecular motifs for the two polymorphs results in comparable interaction energy scales and hence a common thermal response.

From a structural viewpoint, the most significant effect of temperature is to induce changes in the separation between $\mathrm{Ag}^{+}$ cations connected by argentophilic Ag...Ag interactions. ${ }^{[16]}$ The Ag...Ag distance corresponds to the $a$ and $b$ parameters for the trigonal and orthorhombic modifications, respectively. Consequently, the expansivity of these argentophilic interactions is either $97(3)$ or $76.5(8) \mathrm{MK}^{-1}$, corresponding to $\sim 1 \%$ change in length for each $100 \mathrm{~K}$ change in temperature. We note that the Ag...Ag separations in both polymorphs $(\sim 3.6 \AA)$ reflects a particularly weak argentophilic interaction between the $d^{10}$ centres. ${ }^{[16]}$ The increased length of the $\mathrm{Ag} . . . \mathrm{Ag}$ separation in these structures compared to other silver(I) MOFs ${ }^{[17,18]}$ can be explained by the geometric requirement in $\mathrm{Ag}(\mathrm{dca})$ for this separation to equal that of the amide $\mathrm{N}$ centres of neighbouring dicyanamide anions. The key point here is that the flexible structure of both $\mathrm{Ag}(\mathrm{dca})$ polymorphs allows strong PTE effects involving $\mathrm{Ag}$...Ag separations, providing a mechanism by which this system might exhibit a strong spectroscopic sensitivity to temperature.

The excitation spectrum of orthorhombic $\mathrm{Ag}(\mathrm{dca})$ as a microcrystalline powder, measured at $100 \mathrm{~K}$ by monitoring the broad emission maximum at $470 \mathrm{~nm}$, is shown in Fig. 2(a). At high energies, in the deep UV, there is a strong charge transfer band, but at least four additional transitions are identifiable in the wavelength range $200<\lambda<350 \mathrm{~nm}$. The measured photoluminescence is assumed to be phosphorescence, and the presence of several bands in the excitation spectrum indicate that several silver centred optical transitions are allowed. The alternate explanation requires that multiple $\mathrm{Ag}$-species are emissive, however only one Ag site exists in the structure. Ultimately, in the absence of robust time-resolved data, we refrain from attempting any assignment of the four absorbing transitions. The band at $230 \mathrm{~nm}$ shows good temperature stability and was selected for subsequent phosphorescence studies. Following excitation at $230 \mathrm{~nm}$, we observed a broad emission band centred at $470 \mathrm{~nm}\left(21,250 \mathrm{~cm}^{-1}\right)$. The band is wider than expected, extending over the wavenumber range from 15,000 to $25,000 \mathrm{~cm}^{-1}$ [Fig. 2(b)]. The emission energy and band shape is consistent with that of related argentophilic systems-e.g. $\mathrm{TI}\left[\mathrm{Ag}(\mathrm{CN})_{2}\right], \mathrm{K}\left[\mathrm{Ag}(\mathrm{CN})_{2}\right] / \mathrm{KCl}$ and $\mathrm{La}\left[\mathrm{Ag}(\mathrm{CN})_{2}\right]_{3}-$ which show emission in the range from 20,000 to $28,000 \mathrm{~cm}^{-1}$. ${ }^{[18}$, 19, 20] For these cyanide materials the $\mathrm{Ag}$... Ag distances are shorter than in $\mathrm{Ag}(\mathrm{dca})$, which explains the modest shift in emission frequencies. We also observe an extremely large Stokes' shift $\left(15,000 \mathrm{~cm}^{-1}\right)$, which is comparable to that observed for $\mathrm{TI}\left[\mathrm{Ag}(\mathrm{CN})_{2}\right]\left(10,000 \mathrm{~cm}^{-1}\right.$; Ref. [18]). Indicative of highly distorted excited states, the large Stokes' shift can suggest the formation of an exciplex complex, as occurs in the $\mathrm{TI}\left[\mathrm{Ag}(\mathrm{CN})_{2}\right]$ system. ${ }^{[18]}$ Alternatively, the higher energy may be due to a larger energy splitting of the weaker bond formed in the agentophilic interaction. We make no attempt to characterize the nature of the transition (exciplex vs ${ }^{3} \mathrm{dd}$ ), instead focusing solely on the temperature dependence of the emission profile.
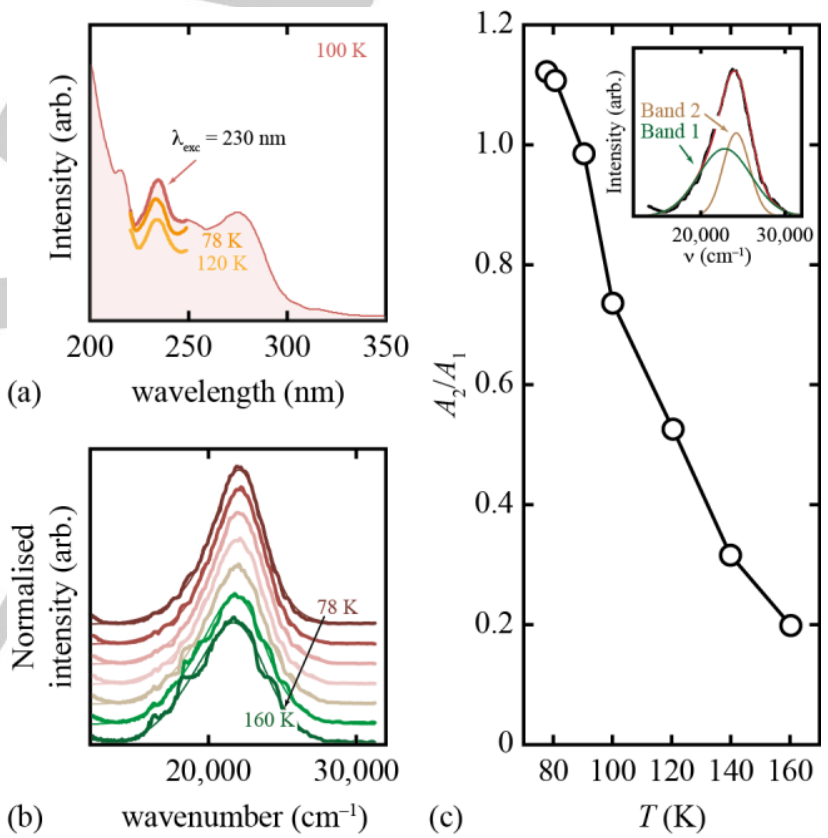

Figure 2. (a) Time-gated excitation spectrum of $\mathrm{Ag}(\mathrm{dca})$ at $100 \mathrm{~K}$ monitoring the emission at $470 \mathrm{~nm}$. Four transitions are evident, superposed on the tail of a charge-transfer transition at higher energies. All time-gated emission spectra were recorded following $230 \mathrm{~nm}$ excitation; this wavelength was chosen because of its good temperature stability. (b) Variable-temperature time-gated emission spectra of $\mathrm{Ag}(\mathrm{dca})$ measured following $230 \mathrm{~nm}$ excitation. The spectra have been background subtracted and the intensities renormalized to give constant-area profiles; successive data sets are shifted vertically for clarity. Experimental data are shown in bold lines, with empirical fits shown in thin lines. (c) Variation in relative area for the two Gaussian components to the empirical fit as a function of temperature, the insert show data and fits [components themselves shown in the inset for the $100 \mathrm{~K}$ data]

By carrying out measurements over the temperature range 78-160 K, we observed a continuous variation in the emission peak profile [Fig. 2(b)]. The emission maximum undergoes a blueshift with increasing temperature, and the asymmetry of the band decreases with increasing temperature. We quantified 
these spectral changes by resolving the band at each temperature using two Gaussian components. The bands can be resolved using two identical Gaussian functions at all temperatures [inset to Fig. 2(c)]. This allows us to conclude that two emissive states are present in $\mathrm{Ag}(\mathrm{dca})$. Band 1 with a maximum at $21,118 \mathrm{~cm}^{-1}$ and a width of $3,850 \mathrm{~cm}^{-1}$, and Band 2 with a maximum at $22,200 \mathrm{~cm}^{-1}$ and a width of $2,140 \mathrm{~cm}^{-1}$. The width of the bands suggest that Band 1 corresponds to a delocalized state or charge transfer state, whereas Band 2 likely originates from a transition in a localized electronic state. We note there is only one $\mathrm{Ag}$ environment in the crystal structure, which allows us to exclude the possibility of excimer formation as this requires at least one distinctly different site in the structure. The temperature dependence of the emission profile is then revealed in progressive changes in the relative area of the two emission bands [Fig. 2(c)]. There is no shift in emission energy of the bands due to the lengthening of $\mathrm{Ag}$....Ag distances from thermal expansion effects, but Band 2 essentially disappears at elevated temperatures. Thus, Band 2 may be due to an optical transition centered in the argentophilic bond, while Band 1 is an intrinsic emission of $\mathrm{Ag}(\mathrm{dca})$. Remarkably, we find that the ratio between the areas of Band 2 and Band 1 shows an essentially linear dependence on temperature, which represents the key optical response of $\mathrm{Ag}(\mathrm{dca})$ over this temperature range. The same variable temperature behaviour was observed following excitation at $270 \mathrm{~nm}$. Likewise, we repeated our measurements for different samples and found no material differences in the observed behaviour.

The optical properties are clearly sensitive even to relatively small changes in the structure of $\mathrm{Ag}(\mathrm{dca})$, as our thermal expansion measurements suggest this distance changes by around $0.029 \AA$ over the range $78-160 \mathrm{~K} .{ }^{[18]}$

\section{Conclusions}

So our results show how the mechanical flexibility of a MOF can be coupled to an optical response. In carrying out this study, we report for the first time the thermal expansion behaviour of $\mathrm{Ag}$ (dca). We find that both trigonal and orthorhombic polymorphs exhibit similar thermal expansion behavior, with NTE along the direction chain axis and extremely strong PTE in the plane that lies perpendicular to this axis. Importantly, this plane includes the argentophilic interactions which govern luminescence behaviour. Consequently the structural changes induced by thermal expansion can have a clear spectroscopic signature, showing how systems such as $\mathrm{Ag}(\mathrm{dca})$ might eventually be exploited in sensing devices. We have also measured the luminescence behaviour of $\mathrm{Ag}(\mathrm{dca})$ and have discovered a systematic shift in emission profile with temperature. We anticipate that this same material is likely to exhibit similar response under application of pressure.

\section{Experimental Section}

Synthesis. All reagents were obtained from commercial suppliers. $\mathrm{Ag}\left[\mathrm{N}(\mathrm{CN})_{2}\right]$ single crystals were grown using the slow diffusion approach.
Saturated aqueous solutions of $\mathrm{AgNO}_{3}(\sim 50 \mathrm{mg})$ and a stoichiometric quantity of $\mathrm{NaN}(\mathrm{CN})_{2}$ were transferred to alternate sides of a glass $\mathrm{H}$-cell which was subsequently filled with distilled water. The system was sealed and left undisturbed until the trigonal polymorph of $\mathrm{Ag}$ (dca) precipitated as single crystals. The orthorhombic polymorph was isolated by direct precipitation from the same reagents. Polycrystalline samples prepared in this way were washed $\left(\mathrm{H}_{2} \mathrm{O}\right)$ and dried under vacuum, and their identity confirmed by powder $\mathrm{X}$-ray diffraction measurements.

Variable-temperature single-crystal $X$-ray diffraction. Single crystal XRD measurements were performed using an Oxford Diffraction SuperNova (Mo Ka) fitted with an Oxford Cryosystems cryostream. ${ }^{[21]}$ The crystal was mounted on a glass fibre and data collections were carried out at intervals of $10 \mathrm{~K}$ across the range $100-350 \mathrm{~K}$. The data were integrated and corrected for absorption using CrysAlisPro, the structure was solved in $P 3_{1} 21$ using ShelXS ${ }^{[22]}$ and the $100 \mathrm{~K}$ data and refined by full-matrix least squares using CRYSTALS. ${ }^{[23]}$ ROTAX was used to identify and implement a transformation matrix, relating the two aligned twinned domains via a 2 -fold rotation about the $z$-axis. ${ }^{[24]}$ Full structural data have been submitted to the CCDC as numbers 1478136-1478141.

Variable-temperature powder $\mathrm{X}$-ray diffraction. Powder $\mathrm{X}$-ray diffraction patterns were measured in a Siemens D5000 (Cu Ka $\left.{ }_{1}, \mathrm{Ka}_{2}\right)$ fitted with an Anton Paar HTK 1200 furnace operating under a helium gas environment. ${ }^{[21]}$ Approximately $80 \mathrm{mg}$ of polycrystalline orthorhombic $\mathrm{Ag}(\mathrm{dca})$ was mixed thoroughly with alumina $(10 \mathrm{mg})$ and silicon powder (10 mg), which acted as internal standards. The powder mixture was carefully loaded onto a stationary sample holder coated with Vaseline. Diffraction patterns were collected at intervals of $10 \mathrm{~K}$ on heating from $303-433 \mathrm{~K}$ and at intervals of $15 \mathrm{~K}$ on cooling from $433-303 \mathrm{~K}$ over the $2 \theta$ range $9^{\circ}-80^{\circ}$. The sample was then rapidly heated to $473 \mathrm{~K}$ and to further scans were measured. Scan times were up to 3 hours with a step size of $0.015^{\circ}$. Rietveld refinements were performed using TOPAS Academic. ${ }^{[25]}$

Variable-temperature photoluminescence. All photoluminescence studies were performed using a Perkin-Elmer LS55 luminescence spectromete in a L-configuration. The standard sample holder was replaced by a customised Oxford Instruments bath cryostat with digital temperature control. Powdered samples of $\mathrm{Ag}(\mathrm{dca})$ were loaded into cuvettes and placed in the cryostat. The time-gated emission spectra were recorded using a delay time of $0.1 \mathrm{~ms}$, a gate time of $10 \mathrm{~ms}$, and excitation and emission slits of $10 \mathrm{~nm}$

\section{Acknowledgements}

A.L.G. gratefully acknowledges financial support from the E.P.S.R.C. (grant EP/G004528/2) and the E.R.C. (Grant 279705). This project has recieved funding from the European Union's Horizon 2020 research and innovation programme under the Marie Sklodowska-Curie grant agreement No. 641887 (Project Acronym: DEFNET-ETN).

Keywords: thermal expansion $\cdot$ argentophilic interactions $•$ luminescence thermochromism • metal-organic frameworks

[1] a) A. L. Goodwin, Phys. Rev. B 2006, 74, 134302. b) J. M. Ogborn, I. E. Collings, S. A. Moggach, A. L. Thompson, A. L. Goodwin, Chem. Sci. 2012, 3, 3011-3017. c) A. Schneemann, V. Bon, I. Schwedler, I. Senkovska, S. Kaskel, R. A. Fischer, Chem. Soc. Rev. 2014, 43, 6062 6096. d) L. Sarkisov, R. L. Martin, M. Haranczyk, B. Smit, J. Am. Chem. 
Soc. 2014, 136, 2228-2231. e) F.-X. Coudert, Chem. Mater. 201527 1905-1916.

[2] S. Horike, S. Shimomura, S. Kitagawa, Nature Chem. 2009, 1, 695-704.

[3] C. Serre, C. Mellot-Draznieks, S. Surblé, N. Audebrand, Y. Filinchuk, G. Férey, Science 2007, 315, 1828-1831.

[4] J. A. Mason, J. Oktawiec, M. K. Taylor, M. R. Hudson, J. Rodriguez, J. E. Bachman, M. I. Gonzalez, A. Cervellino, A. Guagliardi, C. M. Brown, P. L. Llewllyn, N. Masciocchi, J. R. Long, Nature 2015, 527, 357-361.

[5] C. Yang, X. Wang, M. Omary, Angew. Chem. Int. Ed. 2009, 48, 1-6.

[6] W. Li, M. R. Probert, M. Kosa, T. D. Bennett, A. Thirumurugan, R. P. Burwood, M. Parinello, J. A. K. Howard, A. K. Cheetham, J. Am. Chem Soc. 2012, 134, 11940-11943.

[7] S. J. Hunt, M. J. Cliffe, J. A. Hill, A. B. Cairns, N. P. Funnell, A. L. Goodwin, Cryst. Eng. Comm. 2015, 17, 361-369.

[8] a) D. Britton, Y. M. Chow, Acta Cryst. B 1977, 33, 697-699. b) D. Britton, Acta Cryst. C 1990, 46, 2297-2299.

[9] K. Kihara, Eur. J. Miner. 1990, 2, 63-78.

[10] A. B. Cairns, J. Catafesta, C. Levelut, J. Rouquette, A. van der Lee, L. Peters, A. L. Thompson, V. Dmitriev, J. Haines, A. L. Goodwin, Nature Mater. 2013, 12, 212-216.

[11] A. B. Cairns, A. L. Goodwin, Phys. Chem. Chem. Phys. 2015, 17, 20429-21020.

[12] M. A. Rawashdeh-Omary, M. A. Omary, G. E. Shankle, H. H. Patterson, J. Phys. Chem. B 2000, 104, 6143-6151.
[13] S. J. Hibble, G. B. Wood, E. J. Bilbé, A. H. Pohl, M. G. Tucker, A. C Hannon, A. M. Chippindale, Z. Krist. 2010, 225, 457-462.

[14] M. J. Cliffe, A. L. Goodwin, J. Appl. Cryst. 2012, 45, 1321-1329.

[15] A. L. Goodwin, M. Calleja, M. J. Conterio, M. T. Dove, J. S. O. Evans, D. A. Keen, L. Peters, M. G. Tucker, Science 2008, 319, 794-797.

[16] H. Schmidbaur, A. Schier, Angew. Chem. Int. Ed. 2015, 54, 746-784.

[17] C. M. Che, M. C. Tse, M. C. W. Chan, K. K. Cheung, D. L. Phillips, K H. Leung, J. Am. Chem. Soc. 2000, 122, 2464-2468.

[18] M. A. Omary, H. H. Patterson, Inorg. Chem. 1998, 37, 1060-1066.

[19] H. H. Patterson, S. M. Kanan, M. A. Omary, Coord. Chem. Rev. 2000 208, 227-241.

[20] C. L. Larochelle, H. H. Patterson, Chem. Phys. Lett. 2006, 429, $440-$ 444.

[21] J. Cosier, A. M. Glazer, J. Appl. Cryst. 1986, 19, 105-107.

[22] G. M. Sheldrick, Acta Cryst. A 2008, 64, 112-122.

[23] P. Betteridge, J. Carruthers, R. I. Cooper, K. Prout, D. J. Watkin, J. Appl. Cryst. 2003, 36, 1487.

[24] R. I. Cooper, R. O. Gould, S. Parsons, D. J. Watkin, J. Appl. Cryst 2012, 35, 168-174.

[25] A. A. Coelho, Coelho Software, Brisbane.

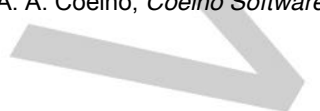


Entry for the Table of Contents (Please choose one layout)

Layout 2:

\section{SHORT COMMUNICATION}

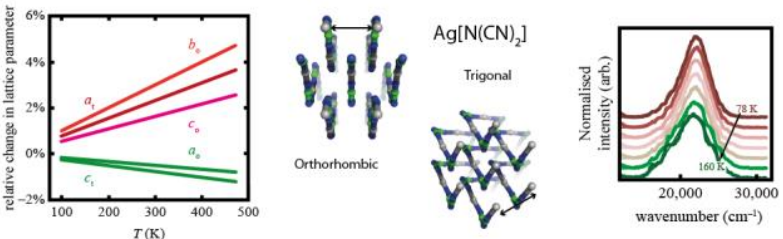

Metal-organic frameworks

Sarah A. Hodgson, Sarah J. Hunt, Thomas Just Sørensen, ${ }^{\star}$ Amber $L$. Thompson, Emily M. Reynolds, Stephen Faulkner, and Andrew L. Goodwin*

Silver(I) dicyanamide exhibits both mechanical and optical sensitivity to temperature. This responsive metal-organic framework may find application in high-precision temperature sensors.

Page No. - Page No.

Anomalous thermal expansion and luminescence thermochromism in silver(I) dicyanamide 


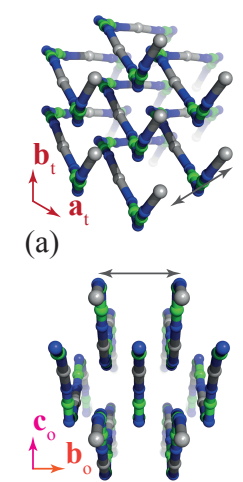

(b)

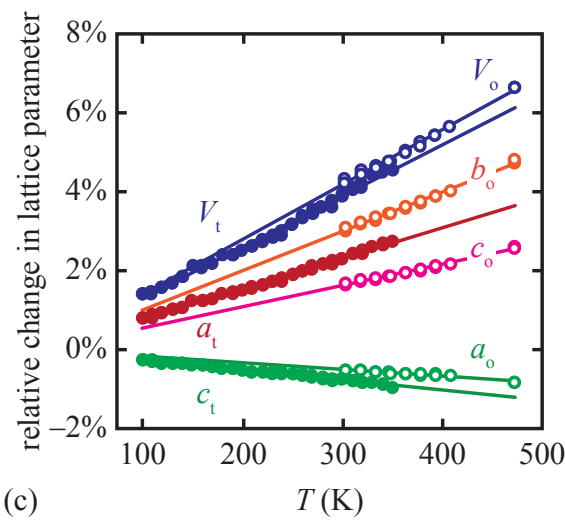

(c)

$T(\mathrm{~K})$ 

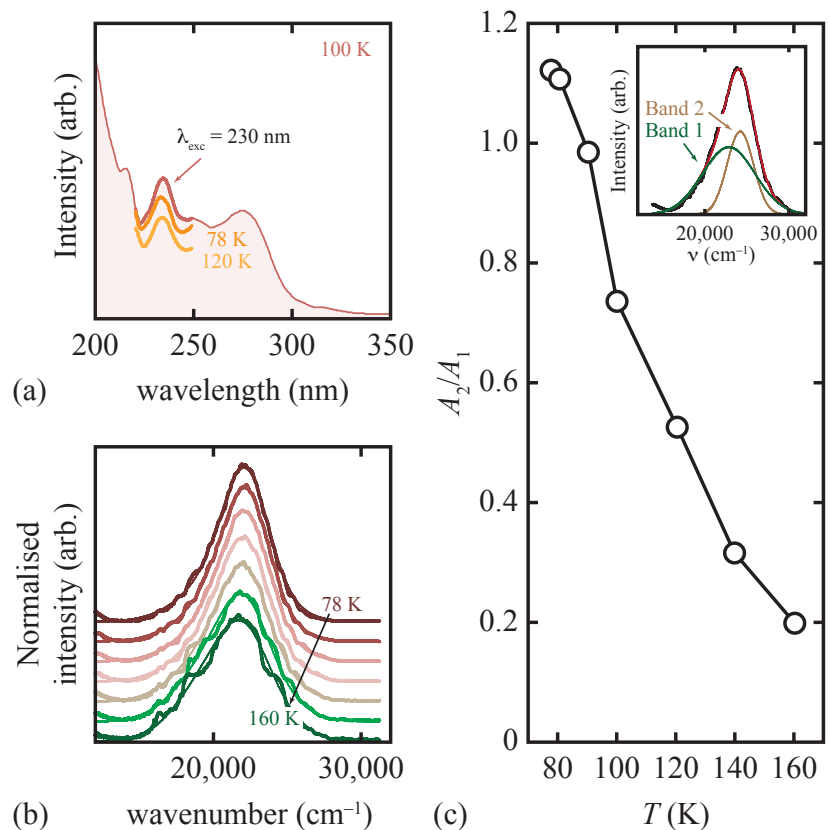